\title{
Impairment of Soft Palate Elevation
}

National Cancer Institute

\section{Source}

National Cancer Institute. Impairment of Soft Palate Elevation. NCI Thesaurus. Code C127225.

An observation of an individual's soft palate elevation during swallowing. 